Research Paper:

\title{
Exploring the Impact of Individual Factors in Taking crossnark Cervical Cancer Screening: A Content Analysis
}

\author{
Vian Haji Rasul', Mohhammad Ali Cheraghi ${ }^{*}$, Zahra Behboodi Moghadam ${ }^{3}$ \\ 1. Department of Nursing, Shaqlawa Technical Institute, Erbil Polytechnic University, Erbil, Iraq. \\ 2. Department of Critical Care Nursing, School of Nursing and Midwifery, Tehran University of Medical Sciences, Tehran, Iran. \\ 3. Department of Reproductive Health, School of Nursing and Midwifery, Tehran University of Medical Sciences, Tehran, Iran.
}

Citation: Haji Rasul, V., Cheraghi, M. A., \& Behboodi Moghadam, Z., 2016. Exploring the Impact of Individual Factors in Taking Cervical Cancer Screening: A Content Analysis. Journal of Client-Centered Nursing Care, 2(4), pp. 239-248. https://doi.org/10.32598/jccnc.2.4.239

: https://doi.org/10.32598/jccnc.2.4.239

Article info:

Received: 11 Apr. 2016

Accepted: 16 Jul. 2016

Keywords:

Cervical cancer

screening, Content analysis, Qualitative research

\begin{abstract}
A B S T R A C T
Background: Cervical cancer remains the leading cause of cancer death among women in developing country. In Iraqi Kurdistan Region, few women undergo screening and the incidence of cervical cancer is on the increase as is the case in most developing countries. The purpose of this study was to explore women's individual factors affecting the performance of the cervical cancer screening in Kurdistan Region, Iraq.

Methods: The present study adopted a qualitative design based on conventional content analysis approach. Purposive sampling was used to select 22 women who had either had a Pap-Smear or refused to have one. Semi structured in depth interviews were conducted with each individual to collect data. Data were collected from August 7, 2015 to July 22, 2016. The criteria for including samples for this study were aS follows: 1) Married Kurdish women who are living in Kurdistan Region, Iraq, 2) Willingness to participate in the study, and 3) Not undergone cervical cancer screening. Non Iraqi Kurdish women were excluded. Each interview lasted for 42-70 minutes on average. In order to analyze the collected data, the obtained purified codes were compared and simultaneously clustered and classified under certain themes using their similarities and differences.
\end{abstract}

Results: Seven main themes including age, educational level, having multiple full-pregnancies, existence of signs and symptoms of cervical cancer, psychological factors, poverty, and marital status emerged during the data analysis.

Conclusion: The findings of this study indicated that individual factors can prevent women from cervical cancer screening in Kurdistan Region, Iraq. Understanding these factors may lead to the development of effective programs and policies to improve cervical cancer screening uptake by Kurdish women.

\section{* Corresponding Author:}

Mohhammad Ali Cheraghi, PhD

Address: Department of Critical Care Nursing, School of Nursing and Midwifery, Tehran University of Medical Sciences, Tehran, Iran. Tel: +98 (21) 66933600

E-mail: mcheraghi@tums.ac.ir 


\section{Background}

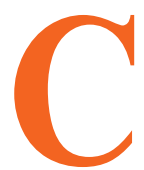

ervical cancer is the second most common cancer in women living in less developed regions (World Health Organization 2015). According to the research findings, cervical cancer has a multi-factorial context. In Kurdistan, some of these factors have been identified; marriage under the age of 18 years, early age at first pregnancy, multiparity, low socioeconomic status, and cervical infections. Also, a study was carried out to detect the prevalence rate of Human papilloma virus (HPV) infection among women with different cervical lesions in Iraqi Kurdistan region showed HPV type 16 was the most common type detected among the positive studied cases (Ali 2010). These factors together with many environmental and epidemiological changes, which the Kurdistan region has been exposed to, could predispose the region to an increased risk of cancer. Cervical cancer screening has proved to be a great step towards cancer prevention and according to World Health Organization (2015), if this screening is done in each area for 10 years, it will decrease the incidence of cervical cancer by $64 \%$ (Beydag 2011).

A Pap-Smear is a simple, quick, and relatively painless screening test for cervix cancer screening. It is aimed at detecting cervical cancer in its early stages, and could reduce both incidence and mortality rates of invasive cervical cancer. Moreover, Pap-Smear coverage discrepancies noted within countries where developing countries has lower coverage (19\%) than developed countries (63\%) (Gakidou, Nordhagen \& Obermeyer 2008). Unfortunately, World Health Organization (2015) reported that there was no data available about estimated coverage of cervical cancer screening in Iraq. However, according to Ministry of Health in Kurdistan Region, nearly 3000 Pap-Smear tests had been done in 2014. Kurdistan Region is an autonomous region in Northern Iraq with a population of 8.35 million (Anon 2016). Although, the Ministry of Health reported an increasing number of women who were referred every year, this number still indicates a few people who have gotten Pap test.

The main aim of cervical screening program is to increase the level of participation and acceptance of cervical screening. However, this must be done in the context of informed consent and understanding of the screening tests (Everett et al. 2011). But one of the significant obstacles to the achievement of cervical cancer screening is the non-acceptance of the program by women. Subsequently, the central motivation of cervical screening programs is to increase the level of acceptance and participation of the screening. It seems that keeping track of the cervical screening test is a situation that is embedded in personal and social self. Therefore, understanding the influencing factors associated with the cervical cancer screening is important. This study intends to provide some description and explanation on the individual influencing conditions for keeping track of the cervical cancer screening.

\section{Materials \& Methods}

\section{Study design}

A qualitative method with content analysis approach was used to describe the experiences and viewpoints of the participating women (Elo \& Kyngas 2008). We chose content analysis due to its well-established ability to elucidate the trends and patterns of communication by systematically coding and categorizing approach. It can be used to unobtrusively explore a large volume of textual information (Gbrich 2007).

\section{Study participants}

Participants consisted of 22 women from Kurdistan Region who were willing to participate in the current research. The participants were chosen based on purposeful sampling strategy according to maximum variation technique. The criteria for including samples for this study were a follows: 1) married Kurdish women who are living in Kurdistan Region, Iraq, 2) willingness to participate in the study, and 3) not undergone cervical cancer screening. Non Iraqi Kurdish women were excluded.

\section{Data collection}

Data were collected from August 7, 2015 to July 22, 2016. Semi-structured in-depth interviews were conducted with each participant. The participants had either taken or refused a Pap-Smear. Interview questions were:

\section{Did you participate in Pap testing?}

Please tell me about individual factors or conditions influencing you to follow /not to follow cervical cancer screening?

The interviews were conducted in a private room in the Pap-Smear center, face to face and individually. Each interview lasted for 42-70 minutes on average. Recruitment continued as long as new themes emerging from the interviews. Data saturation was reached at 19th interview and on the 20th, 21st, and 22nd no extra information was revealed. All interviews were carried out in Kurdish language by the researcher and then 
translated into English. The English texts were revised by a bilingual editor to eliminate any mistranslations. All interviews were recorded by a digital voice recorder and transcribed verbatim at the same day and then analyzed concurrently (Graneheim \& Landman 2004; Speziale, Streubert \& Carpenter 2011).

\section{Data analysis}

In order to analyze the collected data, the transcriptions were carefully reread several times and divided into some manageable, condensed pieces. These data pieces were then abstracted and labeled with codes. The obtained purified codes were compared and simultaneously clustered and classified under certain themes using their similarities and differences. A number of themes were ultimately identified (Graneheim \& Lundman 2004).

\section{Trustworthiness}

The researcher tried to establish credibility, dependability, conformability, and transferability that describe operational techniques supporting the rigor of the work (Lincoln \& Guba 1985). Therefore to establish rigor, this study used prolonged engagement, persistent observation, member check and external check, searching for confirming and disconfirming evidence, researcher credibility, panel of experts, clarity and stepwise replication, constant comparative analysis, maximum variation of sampling. The researcher repeatedly check the data and the participant's talk and with considering the bias and rapid prejudice to provide creation in analysis and credit of data.

\section{Ethical considerations}

The research project was approved by the Ethics Committee of Tehran University of Medical Sciences (project No. 130/1117, approval date: August 10, 2014) and the Ministry of Health and Director of Health in Erbil (No. 65, date: May 12, 2014). Before study, the participants were provided with a detailed description of the study and its risks and benefits during an initial phone contact. The women were ensured about the confidentiality of data then premised to tape-record the interviews and asked to provide informed consent.

\section{Results}

\section{General characteristics of the participants}

The mean age of the respondents was 40.3 years (range: 25-57 years). The married subjects aged 14-28 years. About half (53.3\%) of the participants lived in ur- ban areas and the remaining $(46.7 \%)$ in rural areas. The education level of the respondents ranged from illiterate to doctorate degree. Nearly half of the participants were employed. Moreover, most (80\%) participants were married, one $(6.7 \%)$ was divorced, and two $(13.3 \%)$ were widowed.

\section{Themes}

The results of the study revealed some individual factors and conditions related to cervical cancer screening in Kurdistan region. Seven main themes were age, educational level, having multiple full-pregnancies, existence of signs and symptoms of cervical cancer, psychological factors, poverty, and marital status.

Age

According to the recommendations in the latest guideline of WHO, cervical cancer screening should be applied for women aged 30 years (recommended age to start screening) and older because of their higher risk of cervical cancer. However, the net benefit differs among age groups and may extend to younger and older women depending on their baseline risk of CIN2+ (World Health Organization 2013). In Kurdistan Region, Iraq, however, the recommended age to start cervical cancer screening is three years after marriage to all women according to guideline of Kurdistan Ministry of Health. Because early marriage was common (from 12 years age) in the Kurdish society and according to the latest guideline of World Health Organization (2015), women who were younger than 17 years when they had their first full-term pregnancy are almost 2 times more likely to develop cervical cancer later in life than women who waited to get pregnant until they were 25 years or older. The results of the study showed that just a few number of participants knew the time of the beginning of screening and its regular repetition, and most of them did not exactly know when they should start it and how to follow.

"I know screening tests need to be carried out at younger ages (in fact after marriage) and after it, they are conducted every three years, and if their results are normal. They should be considered." (Participant 1)

"As far as I remember, I think when women exceed 40, they're better to have such test once a year." (Participant 5)

Most of the participants considered conduction of the test necessary for older women or making decision to take the test after the age of 40 . One of the women had 
the test at her 40 for the first time, she explained the reason for her selection of this age as:

"I knew if there were any problems before that age, they would be at their early phases. After that age; however, if there is a problem, it will be so serious and it can be too late for treatment. That is why I told myself that I needn't sit on my hands and I need to make a serious decision.'(Participant 1)

Among younger women that considered themselves to be away from developing cervical cancer, some thought that conduction of the test was unnecessary for them while some agreed with its necessity, and some refused to have the test.

A 38-year-old woman who had never had the test said:

"Well, because I am young, I think that when a woman gets old, need to carry out preventive tests." (Participant 15)

A 27-year-old participant who had frequent infections said:

"I'm sure that the result will be negative, that is why I'm not worried about it. I'm sure I've got no problems, it's too early for me to get this disease. By conducting the test, I'm quite sure, and I'll be happy and feel comfortable." (Participant 11)

A 34-year-old participant who refused to have the test said:

"The symptoms of this disease don't emerge until 10-15 years, and I'm young and don't have any signs. God is great, who knows what will happen by then and what my destination will be. It's possible that I won't be alive by then, who knows who's dead or alive." (Participant 6)

\section{Educational level}

The women who participated in this study had different educational levels from illiterate to doctorate. This study indicated that educational level did not affect the cervical cancer screening.

A 48-year-old participant who had finished fifth grade of elementary school said: "Now that doctor has advised me that this test is good for my health and control, I believe it's very important, and this motivates me to go for it once my appointment is due or even before that." (Participant 9)

On the opposite, a woman who held a bachelor's degree in midwifery explained her reason for refusing to have Pap test as follows:
"This may be attributed to the fact that we don't go to doctor unless some problems happen to us. Unfortunately, even those who work in the health field know what science says but they're also too lazy to take it seriously." (Participant 5)

The results of the study showed that the educational level did not seem to be correlated with eagerness of what Pap test is used for and meant to. There is no clear relationship between taking action and being inactive to have screening test, and there are ambiguities almost among women with different levels of education who need necessary information. In this regard, a graduate in Agriculture major said:

"When the doctor talked to me about this test, it was quite new to me, and something formed in my mind that affected me. Now I sometimes think about it, and I'd like to have the test." (Participant 6)

Even if, the women at the lowest educational level like to have a Pap test; if they knew that a Pap test was used for cervical cancer screening.

An illiterate participant who was familiarized with the test after she had referred to doctor talked about the reason for having the test like this:

"I was really scared of receiving a positive result, and then I made my mind to have the test. I would thought to myself: 'If I have it, it's better for me to know about it as soon as possible. It's better for me, and the treatment will be easier.' Then I referred to the Health Center and had the test."(Participant 3)

Therefore, educational level is not a barrier to know what Pap-Smear is used for and why it is important to have a Pap-Smear and health managers related to subject in Kurdistan Region should improve the cervical cancer screening programs. For example, newspapers, magazines, TV and the radio are different ways for women with low and high educational women to get the knowledge that Pap-Smear is a test used for screening.

\section{Having multiple full-term pregnancies}

According to the latest guideline of WHO, women with 3 or more full-term pregnancies have higher risk of developing cervical cancer.

The researcher tried to include women with different number of children ( 0 to 10$)$ in the study. However, none of the participants talked about the relationship between 
the number of their children and higher probability of developing cervical cancer, instead they were concerned about their children if developing the disease, and that was one of the reasons for referring for the test. A participant who had two children talked about her main motive for having the test as:

"Staying alive is my main motive, because when I look at myself, I have two small kids, and if I'm not there for them, what will happen to them? That is why I want to stay alive by all means, it is for my kids in the first place." (Participant 12)

Another participant who had 10 children talked about her main reason for having Pap test as this:

"Because my kids were small and their dad was dead, I kept thinking if I'm not with them, what will happen to them. Now my children are deprived from having father, and if I die, they'll become complete orphans. I was thinking of this issue for some time, and I started to believe that I needed to refer to a doctor, and if I'm inflicted, it's better to know about it sooner." (Participant 18)

Existence of signs and symptoms of cervical cancer

Women with early cervical cancers and pre-cancers usually have no symptoms. Symptoms often do not begin until a pre-cancer becomes a true invasive cancer and grows into nearby tissue. When this happens, the most common symptoms are abnormal vaginal bleeding, an unusual discharge from the vagina, and pain during sexual relationship (vaginal intercourse). Therefore, women would be better not to wait for symptoms to appear and be screened regularly.

The majority of the participants highlighted the necessity of a Pap test when signs and symptoms were present. They believed that under such circumstances, a woman might be at risk of developing cancer. Women with no problem considered the test unnecessary and decide not to do it.

"I told I didn't have any problems, and why I should have the test. I've never thought about the Pap test for myself." (Participant 8)

These common signs and symptoms of cervical cancer can also be caused by other conditions other than cervical cancer like an infection (which can cause pain or bleeding).

According to the latest guideline of World Health Organization (2015), if women have any of these problems, they should see their health care professional right away - even if they have been getting regular Pap tests. If it is an infection, it will need to be treated. If it is cancer, ignoring symptoms might allow it to progress to a more advanced stage and lower their chance for effective treatment.

\section{Psychological factors}

Women subconsciously referred to their psychological factors to approach different life situations like cervical cancer screening. Being frightened to refer to doctor and have necessary tests is considered as one of the mental factors that causes some participants to refuse taking the test although they know about its necessity and importance. Therefore, it is difficult for them to make their minds, so they avoid the test. A 48-year-old participant said:

"When the doctor told me that I needed to have this test at the Maternal and Infant Hospital, it took me one week to make my mind and to get over my fear. I'm a coward, and I get frightened by any test that is recommended to me. For example, once I had a toothache, and I was scared to go to dentist. I believe this test is like other tests for me, and that's why I'm scared." (Participant 9)

Another participant who refused to have the test talked about her fear to have the test this way,

"In the first place, I'm really scared of both the test and its result. I'm a very fearful person. Believe me, I didn't dare to insert IUD while everyone says it's not painful at all. I prefer to prevent pregnancy through other ways. I'm that much scared of both the test and the result." (Participant 17)

On the other hand, some participant referred to their bravery while going to their doctor.

"Whenever I get sick, I visit my doctor, and I'll follow all her orders whether she prescribes me drugs or examinations. I'm brave for such things. For me, this test was not different from other tests. I know whatever my doctor advises me is good for my health, so I follow her orders." (Participant 10)

Discomfort emerged as another subtheme in this study. Due to the discomfort during gynecological tests (with the insertion of the speculum), women were reluctant to be tested. One participant reported:

"Generally, I do not like gynecological tests, especially when the speculum is used, and I do not want to have them unless I am forced to." (Participant 13) 
A few participants suggested embarrassment and shyness as the reasons for their refusal. One of the participants explained:

"My main problem is the shame of the Pap test. Every time, I think to have a Pap test I change my mind because I feel embarrassed if someone does the test for me." (Participant 4)

However, the majority of the participants did not describe embarrassment as a major barrier since the test was performed by a female healthcare provider.

Some participants expressed feelings of unsafety and anxiety caused by their mistrust of hospitals. Such mistrust prevented them from having a Pap test even if they decided to have one. The participants did not trust in the sampling instruments, the individuals who collected the samples, or the laboratory results. A participant explained:

"Facilities and equipment used in the hospital for my test were a barrier. I was afraid that dirty and unsterile equipment might be used." (Participant 1)

Another woman who refused the test stated:

"If I want to go and do the test, I doubt the laboratory and I will not be sure that the result is correct." (Participant 5)

\section{Poverty}

Pap test is conducted for free in governmental centers; therefore, financial conditions do not affect the test conduction much. The participants had a monthly income of about 300 to 3500 dollars; however, women with low and average incomes stated that if the test were expensive and were carried out outside the country, they would not be able to have it.

"In fact, life should let us refer for the test, if the test is expensive, I can't have it." (Participant 13).

Poverty is also a risk factor for cervical cancer. Many women with low income do not have immediate access to adequate health care services, including Pap tests. This means they might not get screened or treated for cervical cancers and pre-cancers. Some participants stated that although the test was conducted for free in governmental centers, they were worried about the expenses of cancer treatment, which was one of the causes for refusing to have the test.

"I think fear of treatment can also cause us to neglect having the test. When I think to myself and say suppose I go and have the test, and I come to know that I have cancer, then how can I treat it and where can I bring the money to buy the drugs?" (Participant 5)

\section{Marital status}

Participants of the present study consisted of married, divorced, and widowed women. According to the guidelines of Kurdistan Region health system, all women should have the screening test for cervical cancer after marriage; however, some participants stated that they thought the test is necessary only for those who have a husband. A divorced woman who was advised Pap test due to her abnormal bleeding said:

"When I heard this advice, I was socked in the first place, because I've been separated from my husband for many years, I thought I didn't need Pap test, and I had never thought that this test was also used for divorced women; therefore, I had never thought about it before that time." (Participant 2)

In this part the researcher found individual conditions, which in this research were the immediate set of conditions faced by women on cervical cancer screening.

\section{Discussion}

The individual factors affect cervical cancer screening and play an important role. Individual factors found in this research included age, educational level, multiple full-term pregnancies, signs and symptoms of cervical cancer, psychological factors, poverty, and marital status. Early age of marriage and first pregnancy is one of the known risk factors of cervical cancer that is common in Kurdistan Region. The majority of women who used or did not use cervical cancer screening services were not aware of the start of screening time and most of them believed testing is just necessary for old women. This finding was consistent with the result of other previous studies that reported increasing age was associated with a decreased use of screening services (Nene et al. 2007). Whilst one study showed Zimbabwean women between the ages of 25 and 34 were more likely to access cervical screening compared with those equal or larger than 45 years (Mupepi, Sampselle \& Johnson 2011).

Also another study that mentioned women aged 3544 years were more likely to have ever had a Pap test compared with those aged 45-54 years (Bessler, Aung \& Jolly 2007). In addition, the study participants in South Africa believed that the age for the Pap test should be younger than 20 and should be repeated at five-year intervals (Sibiya \& Grainger 2007). Based on the study results, educational level of Kurdish women did not reflect on the cervical cancer screening and all women regardless of their educational level had low level of knowl- 
edge on the benefits of the test and prevention of cervical cancer and need to receive more information. One study was done in Kenya found that the level of cervical cancer understanding and screening history was low among women with a high level of education (Ngug et al. 2012).

Whilst Bessler, Aung and Jolly (2007) study result in Jamaica showed that education had a strong influence on Pap screening uptake. There was correlation between higher education level and increased cervical cancer screening attendance. Other cross-sectional study among rural women in South Africa mentioned that an important aspect with regard to cervical cancer screening is whether women who have a higher educational background and a better knowledge of cervical cancer screening also have a higher rate of Pap-Smears (Hoque, Hoque \& Kader 2008).

We found that having multiple full-term pregnancies had no effect on cervical cancer screening as our participants were not aware that women with 3 or more full pregnancies had an increased risk for developing cervical cancer. Similarly, a cross-sectional study conducted in Kenya indicated just $10 \%$ of their patricians identified having many children as a risk factor for cervical cancer (Murugl 2014). Also, most Queensland women in a computer assisted telephone interview survey did not consider that high parity could increase the risk of cervical cancer (Christie 2013).

The majority of women performed cervical cancer screening when they had gynecological problems or any abnormal signs and symptoms. However, lack of any symptoms made some women ignore the importance of cancer prevention. Similarly many Korean American women thought that cancer screening tests were needed only after symptoms of cancer occur and they should only seek care when they become sick (Lee, Fogg \& Menon 2008). Fort et al. argued that women who had immediate symptoms of cervical cancer sought screening (Fort et al., 2011). Similar findings were reported by Morema et al. (2014) and Murugl (2014). However the most important risk factor for cervical cancer is infection by the $\mathrm{Hu}$ man Papilloma Virus (HPV) but none of the interviewed women were aware of the connection between HPV, change of cells in cervix, and cervical cancer. This result is consistent with Wong et al. (2009) and Hounsgaard et al. (2013) study results, while different from that of Guilfoyle et al. (2007) and Nene et al. (2007) results.

Psychological status of women was another important barrier as mentioned by some participants. Some women in our study also reported feeling of fear, embarrassment and discomfort during the procedure as other barriers to screening. The exposure of private parts of the body and insertion of the speculum during a pelvic examination were commonly mentioned by the participants. Embarrassment, fear, and pain were also highlighted by older African American and Hispanic women in New York (Guilfoyle, Franco \& Gorin 2007). Similar results were also reported in other studies (Byrd, Chavez \& Wilson 2007; Waller et al. 2009; Menard et al., 2010).

In addition, mistrust of laboratory test results and fear of infection were reported by our participants. Similarly, women in Malaysia were worried about pain and possible contamination during a Pap test (Al-Naggar \& Isa 2010). Mistrust of the smear taker was also another barrier to perform a Pap test in the current study. A study in Canada introduced insufficient familiarity with the care provider and discomfort with the power imbalance between themselves and the care provider as significant barriers to cervical cancer screening among aboriginal women (Maar et al. 2013). Therefore, to increase trust in cervical cancer screening services, the Ministry of Health should train the smear takers and compel the use of disposable instruments. Since psychological barriers are not easy to overcome, effective information provision and communication between women and healthcare providers are necessary. Clear explanation about cervical cancer and a Pap-Smear procedure can help decrease women's anxiety, fear, discomfort, and embarrassment.

Financial status can also play a role on cervical cancer screening. Women with low incomes were less interested in undertaking the screening even though test was done for free in public health centers. The women were worried about the cost of cancer treatment if diagnosed. Sibiya and Grainger (2007) argued that if the cervical screening was provided without any charge it would help more women to use Pap test services. The National Breast and Cervical Cancer Early Detection Program has provided free or low-cost screening and diagnostic breast and cervical cancer services to low-income, underinsured, and uninsured women and access to state Medical programs for treatment (Benard et al. 2014). Cronje and Beyer (2007) reported that coverage of the population chiefly depends on the provision of funds, initiatives on the part of health care services and the compliance of the targeted population. Also the women were concerned about the indirect costs of getting screened, including transportation, visit of doctors, and medicines fees (Fort et al., 2011). Delay in definitive therapy for cervical cancer occurs among women with lower income and educational background (Ashing-Gaiwa \& Rosales 2013). 
According to our participants' opinions, taking Pap test was known necessary and important just for married women who have husbands. However other women like widows or divorced females felt no need for screening based on their relationship status. Because they had no partners they felt there was no risk. These results are consistent with previous study done by Leach and Schoenberg (2007). They reported that married women were $43 \%$ more likely to follow their Pap tests compared with all other women (Leach \& Schoenberg, 2007). Also this was similar finding by Nene et al. (2007) and Marlow et al. (2015).

This study set out to explore in a qualitative research how Kurdish women are involved in cervical cancer screening processes in Kurdistan region, Iraq. According to the study results, individual factors affect cervical cancer screening process. In addition, preventive cares like conducting check-ups and routine tests, including Pap test have not become a part of the Kurdish people's culture. Therefore, the findings of this study suggest that the decision to undertake cervical screening is influenced by a combination of factors. The knowledge about cervical cancer and its screening is essential for taking Pap test.

In this study, we only included Iraqi Kurdish Muslim women who lived in Kurdistan Region of Iraq. The findings of this study have potential implications for the Ministry of Health and health policymakers who are interested in improving cervical cancer screening.

Although the individual factors of women affecting cervical cancer screening were clearly described by women in this study, future research is warranted to elucidate how to improve the cervical cancer screening program in Kurdistan region and to increase the acceptability of the program.

\section{Acknowledgments}

This study was financially supported by International Campus, Tehran University of Medical Sciences, and Erbil Polytechnic University, Erbil Ministry of Higher Education. Therefore, authors thank for all of financial and technical support for doing the project.

\section{Conflict of Interest}

The authors declared no conflict of interests.

\section{References}

Ali K. S., 2010. Molecular and immunological study of human Papilloma virus infections among patient with different cervical lesions in Kurdistan region-Iraq (PhD thesis). Kurdistan: Hawler Medical University.

Al-Naggar, R. A., \& Isa, Z. M., 2010. Perception and opinion of medical students about pap-smear test: A qualitative study. Asian Pacific Journal of Cancer Prevention, 11(2), pp. 435-40. PMID: 20843130

Anon, 2016. Kurdistan at a glance, the Kurdistan Region in Brief, Kurdistan Regional government [Internet], viewed 11 Feb 2016, http://www.gov.krd

Ashing-Giwa, K. \& Rosales, M., 2013. Evaluation of therapeutic care delay among Latina and European-American cervical cancer survivors. Gynecologic oncology, 128(2), pp. 160-5. doi: 10.1016/j.ygyno.2012.11.015

Benard, V. B., et al., 2014. Vital signs: Cervical cancer incidence, mortality, and screening - United States, 2007-2012. Morbidity and Mortality Weekly Report, 63(44), pp. 1004-9. PMID: 25375072

Bessler, P., Aung, M., \& Jolly, P., 2007. Factors affecting uptake of cervical cancer screening among clinic attendees in Trelawny, Jamaica. Cancer Control, 14(4), pp. 396-404. PMID: 17914340

Beydag, K. D., 2011. Knowledge and applications of the midwives and nurses at an educational hospital on the early diagnosis of cervix cancer. Asian Pacific Journal of Cancer Prevention, 12(2), pp. 481-5. PMID:21545217

Byrd, T. L., Chavez, R., \& Wilson, K. M., 2007. Barriers and facilitators of cervical cancer screening among Hispanic women. Ethnicity and Disease, 17(1), pp. 129-34. PMID: 17274222

Christie, L. M., 2013. Factors influencing the impact of primary and secondary prevention strategies for cervical cancer among Queensland women (PhD Thesis). Brisbane: Queensland University of Technology.

Cronje, H. S., \& Beyer, E., 2007. Screening for cervical cancer in an African setting. International Journal of Gynecology $\mathcal{E}$ Obstetrics, $98(2)$, pp. 168-71. doi: 10.1016/j.ijgo.2007.05.005

Elo, S., \& Kyngäs, H., 2008. The qualitative content analysis process. Journal of Advanced Nursing, 62(1), pp. 107-15. doi 10.1111/j.1365-2648.2007.04569.x

Fort, V. K., et al., 2011. Barriers to cervical cancer screening in Mulanje, Malawi: A qualitative study. Patient Prefer Adherence, 5, pp. 125-31. doi: 10.2147/PPA.S17317

Everett, T., et al., 2011. Interventions targeted at women to encourage the uptake of cervical screening. The Cochrane Library, 5. doi: 10.1002/14651858.CD002834.pub2

Gakidou, E., Nordhagen, S., \& Obermeyer, Z., 2008. Coverage of cervical cancer screening in 57 countries: Low average levels and large inequalities. PLoS Medicine, 5(6), p. 132. doi: 10.1371/journal.pmed.0050132

Gbrich, C., 2007. Qualitative data analysis: An introduction. London: Saga Publications.

Graneheim, U., \& Lundman, B., 2004. Qualitative content analysis in nursing research: Concepts, procedures and measures to achieve trustworthiness. Nurse Education Today, 24(2), pp 105-112. doi: 10.1016/j.nedt.2003.10.001. 
Guilfoyle, S., Franco, R., \& Gorin, S. S., 2007. Exploring older women's approaches to cervical cancer screening. Health Care for Women International, 28(10), pp. 930-50. doi: $10.1080 / 07399330701615358$

Hoque, M., Hoque, E., \& Kader, S. B., 2008. Evaluation of cervical cancer screening program at a rural community of South Africa. East African Journal of Public Health, 5(2), pp. 111-6. PMID: 19024420

Hounsgaard, L., et al., 2013. Women's perspectives on illness when being screened for cervical cancer. International Journal of Circumpolar Health, 72(1), p. 21089. doi: 10.3402/ijch. v72i0.21089

Leach, C. R., \& Schoenberg, N. E., 2007. The vicious cycle of inadequate early detection: A complementary study on barriers to cervical cancer screening among middle-aged and older women. Preventing Chronic Disease, 4(4), p. 95. PMCID: PMC2099293

Lee, E. E., Fogg, L., \& Menon, U., 2008. Knowledge and beliefs related to cervical cancer and screening among Korean American women. Western Journal of Nursing Research, 30(8), pp. 96074. doi: $10.1177 / 0193945908319250$.

Lincon Y. S., \& Guba E. G., 1985. Naturalistic inquiry. California: Sage Publications.

Maar, M., et al., 2013. A qualitative study of provider perspectives of structural barriers to cervical cancer screening among first nations women. Women's Health Issues, 23(5), pp. 319-25. doi: 10.1016/j.whi.2013.06.005.

Marlow, L. A. V., Waller, J., \& Wardle, J., 2015. Barriers to cervical cancer screening among ethnic minority women: A qualitative study. Journal of Family Planning and Reproductive Health Care, 41(4), pp. 248-54. doi: 10.1136/jfprhc-2014-101082.

Menard, J., et al., 2010. Barriers to cervical cancer screening among Haitian immigrant women in Little Haiti, Miami. Journal of Cancer Education, 25(4), pp. 602-8. doi: 10.1007/s13187010-0089-7.

Morema, E. N. et al., 2014. Determinants of cervical screening services uptake among 18-49 year old women seeking services at the Jaramogi Oginga Odinga teaching and referral hospital, Kisumu, Kenya. BMC Health Services Research, 14(1). pp. 335. doi: 10.1186/1472-6963-14-335.

Mupepi, S. C., Sampselle, C. M. \& Johnson, T. R. B., 2011. Knowledge, attitudes, and demographic factors influencing cervical cancer screening behavior of Zimbabwean women. Journal of Women's Health, 20(6), pp. 943-52. doi: 10.1089/jwh.2010.2062.

Murugl, N. A., 2014. Determination of cervical cancer screening uptake among women in Embu country, Kenya (MSc. Dissertation). Nairobi: University of Nairobi.

Nene, B., et al., 2007. Determinants of women s participation in cervical cancer screening trial, Maharashtra, India. Bulletin of the World Health Organization, 85(4), pp. 264-72. doi: 10.2471/ blt.06.031195

Ngugi, C. W. et al., 2012. Factors affecting uptake of cervical cancer early detection measures among women in Thika, Kenya. Health Care for Women International, 33(7), pp. 595-613. doi: 10.1080/07399332.2011.646367.

Sibiya, M. N., \& Grainger, L., 2007. An assessment of the implementation of the provincial cervical screening programme in selected primary health care clinics in the llembe region, KwaZulu-Natal. Curationis, 30(1). doi: 10.4102/curationis. v30i1.1050.

Speziale, H. S., Streubert, H. J., \& Carpenter, D. R., 2011. Qualitative research in nursing: Advancing the humanistic imperative. Philadelphia: Lippincott Williams \& Wilkins

Waller, J., et al., 2009. Barriers to cervical cancer screening attendance in England: A population-based survey. Journal of Medical Screening, 16(4), pp. 199-204. doi: 10.1258/jms.2009.009073.

Wong, L. P., et al., 2009. Knowledge and awareness of cervical cancer and screening among Malaysian women who have never had a pap-smear: A qualitative study. Singapore Medical Journal, 50(1), pp. 49-53. PMID: 19224084

World Health Organization, 2013. Early detection of cancer [Internet]. Available from http://www.who.int/cancer/detection/en/

World Health Organization, 2015. Human papillomavirus (HPV) and cervical cancer [Internet]. Available from http://www. who.int/mediacentre/factsheets/fs380/en/ 
Autumn 2016 . Volume 2. Number 4

Client-Centered Nursing Care 